\title{
Two-fluid temperature-dependent relativistic waves in magnetized streaming pair plasmas
}

\author{
A. R. Soto-Chavez, * S. M. Mahajan, and R. D. Hazeltine \\ Institute for Fusion Studies, The University of Texas at Austin, Austin, Texas 78712, USA \\ (Received 26 August 2009; revised manuscript received 1 December 2009; published 10 February 2010)

\begin{abstract}
A relativistic two-fluid temperature-dependent approach for a streaming magnetized pair plasma is considered. Such a scenario corresponds to secondary plasmas created at the polar caps of pulsar magnetospheres. In the model the generalized vorticity rather than the magnetic field is frozen into the fluid. For parallel propagation four transverse modes are found. Two are electromagnetic plasma modes which at high temperature become light waves. The remaining two are Alfvénic modes split into a fast and slow mode. The slow mode is cyclotron two-stream unstable at large wavelengths and is always subluminous. We find that the instability cannot be suppressed by temperature effects in the limit of large (finite) magnetic field. The fast Alfvén mode can be superluminous only at large wavelengths, however it is always subluminous at high temperatures. In this incompressible approximation only the ordinary mode is present for perpendicular propagation. For oblique propagation the dispersion relation is studied for finite and large strong magnetic fields and the results are qualitatively described.
\end{abstract}

DOI: 10.1103/PhysRevE.81.026403

PACS number(s): 52.27.Ny, 52.30.Ex, 95.30.Qd, 97.60.Gb

\section{INTRODUCTION}

Relativistic electron-positron (e-p) plasmas may be a major source of radio emissions emanating from pulsar magnetospheres (see, e.g., [1,2]). The pulsar plasmas are composed of two main ingredients: an ultrarelativistic charged beam (the primary beam) and the secondary relativistic e-p plasma beams [3]. Studies of wave propagation in such plasmas (see, e.g., [4-9], and references therein) have invoked fluid models as well as the kinetic theory. Relativistic fluid models, in general, do not include temperature effects ([4] and references therein) while studies, based on kinetic theory, use artificial particle distribution functions (such as waterbag) to derive dispersion relations [5,7]. Even when more appropriate distributions (such as the Maxwellian) are investigated, the range of temperatures is restricted (too low or too high) [6], and streaming effects are sometimes not taken into account [8].

An important development in the evolution of the study of wave propagation in pulsar plasmas is the recent demonstration [10] that the e-p distribution function is likely to be a Maxwellian with temperatures very close to $T \approx m_{o} c^{2} / k_{B}$, i.e., the average random energy is of the order of the rest mass. It is therefore critical to determine the exact properties of waves in e-p plasmas at such temperatures. For this we investigate a relativistic two-fluid model to study propagation of waves in streaming magnetized pair plasmas. This work is different from previous studies in that the plasma is treated fully relativistically - both in temperature and in directed speed. Among other things, in this paper we attempt to determine, for example, how temperature will affect the results of Verdon and Melrose [9].

\section{Pulsar plasmas properties}

We begin with a brief recapitulation of the pulsar polar cap model (see, e.g., [11] for a complete review). The pair

\footnotetext{
*arsoto@physics.utexas.edu
}

plasma, that populates the polar caps of rotating neutron stars, is probably produced by an electromagnetic avalanchelike process. These rotating (strongly magnetized) neutron stars induce strong electric fields that can rip charges off their surfaces and accelerate them along the magnetic field $B$, creating a very high relativistic beam (primary beam) whose distribution function becomes one dimensional in momentum space (after loosing their perpendicular momentum through synchrotron radiation). The primary beam particles, streaming along $B$, emit curvature radiation photons $[1,3]$ that, in turn, produce pairs through the interaction $\gamma+B$ $\rightarrow e^{-}+e^{+}$, causing a cascade. The secondary (or tertiary) pairs may be born into an excited Landau level with energies $E^{2} / m^{2} c^{4}=1+p^{2} / m^{2} c^{2}+(2 n) B / B_{c r}$ where $p$ is the momentum along $B ; B_{c r}=m^{2} c^{3} / e \hbar$ is the critical magnetic field and $n$ is a nonnegative integer specifying the Landau level $[10,11]$.

The density of the primary beam $n_{b}$ is less than the density of the secondary pair plasma; the latter is assumed to be $n_{ \pm} \sim \Gamma n_{G J}$, where $\Gamma$ is a multiplication factor (not well understood). The reference density $n_{G J}=\boldsymbol{w} \cdot \boldsymbol{B} / 2 \pi e c$, defined in terms of $w=2 \pi / P$ (the rotational frequency of the star), is called the Goldreich-Julian or "corotating" density [12].

The possibility of streaming of this secondary plasma, comes from the requirement that the total charge density of the three components (primary beam plus electrons and positrons of the secondary plasma) must be equal to the charge density of corotation [13]. The secondary pair plasma is assumed to be neutral and since $n_{G J} \propto B \cos \theta$ ( $\theta$ is the angle between $w$ and $B$ ), while it can be shown that $n_{b} \propto B$ [13], a deviation develops between the two as the flow propagates along $B$. Consequently, one type of secondary particles will be accelerated (say electrons) and the other (positrons) will be decelerated to screen out the electric field that could result from that deviation. Implying that in the comoving center of momentum (CM) frame, i.e., the frame that moves with the speed at which the pair was created, the positive and negative streams move in opposite directions $[6,13]$. Therefore a streaming or sometimes called counterstreaming effect will be produced. It is believed that the streaming pair plasma 
contained in near the polar caps is the source of radio pulses that we detect on Earth.

In this paper we study dispersion relations and wave properties in an e-p counterstreaming magnetized relativistic plasma. In Sec. II we introduce the fluid model. In Sec. III we study the model for relativistic waves at three different angles of propagation with respect to the magnetic field (parallel, perpendicular and oblique). We discuss temperature effects on the wave modes and on the instabilities found. In Sec. IV we give some numerical estimates. In Sec. V our results are summarized and we briefly discuss the implications of the unstable mode on radio emission theories.

\section{TWO-FLUID RELATIVISTIC EQUATIONS}

If the particle distribution function of the secondary particles is taken to be a local relativistic Maxwellian, that is $f_{s} \propto \operatorname{Exp}\left(-U_{\mu} P^{\mu} / T_{s}\right)$, then the dynamics of such plasmas are described by the following (see, e.g., [14-17]) relativistic fluid equations coupled to the electromagnetic field via

$$
\partial_{\mu} T_{(s)}^{\mu \nu}=F^{\nu \alpha} j_{\alpha(s)},
$$

where

$$
\begin{gathered}
T_{(s)}^{\mu \nu}=h_{(s)} U_{(s)}^{\mu} U_{(s)}^{\nu}+p_{(s)} \eta^{\mu \nu}, \\
F^{\mu \nu}=\partial^{\mu} A^{\nu}-\partial^{\nu} A^{\mu},
\end{gathered}
$$

are, respectively, the energy-momentum tensor of the fluid species $s$ and the electromagnetic field tensor. Where $U_{s}^{\mu}$ $=\left\{\gamma_{s}, \gamma_{s} \boldsymbol{V}_{s} / c\right\}$ is the fluid four-velocity, $\gamma=\left(1-V^{2} / c^{2}\right)^{-1 / 2}$ is the Lorentz factor and $\eta^{\mu \nu}=(-1,1,1,1) . j_{(s)}^{\mu}=q_{s} n_{R(s)} U_{(s)}^{\mu}$ is the four current, $p_{s}$ the pressure and $h_{s}$ is the enthalpy density per unit volume given by [14]

$$
h_{s}=n_{R} m_{s} c^{2} \frac{K_{3}\left(z_{s}\right)}{K_{2}\left(z_{s}\right)} \equiv n_{R} m_{s} c^{2} G\left(z_{s}\right),
$$

where $K_{j}$ is the modified Bessel function of $j$ kind, with argument $z_{s}=m_{s} c^{2} / T_{s} ; q_{s}, m_{s}, n_{R}$, and $T_{s}$ are, respectively, the invariants: charge, rest mass, rest density and temperature for both species. The relativistic equations of motion (1) can be broken down into their zero and three components. The three or vector component gives (the zero component would not be used),

$$
n_{s} \frac{d}{d t}\left(m_{s} G_{s} \gamma_{s} \boldsymbol{V}_{s}\right)+\nabla p_{s}=q_{s} n_{s}\left[\boldsymbol{E}+\left(\boldsymbol{V}_{s} \times \boldsymbol{B}\right) / c\right],
$$

where $d / d t \equiv \partial / \partial_{t}+\boldsymbol{V}_{s} \cdot \boldsymbol{\nabla}$. Equation (5) shows that when temperature effects are properly included the effective momentum of the charged fluid $s$ becomes $\boldsymbol{P}=m G \gamma \boldsymbol{V}$. Note that $G\left(z_{s}\right)$ is a function of temperature only. We refer the reader to [14] for more details and simple state that, by taking the curl of Eq. (5) it can be cast, after some algebra, in a two-fluid vortex dynamical form (or equivalently by taking the curl of Eq. (36) in Ref. [14])

$$
\frac{\partial \Omega_{ \pm}}{\partial t}=\nabla \times\left(\boldsymbol{V}_{ \pm} \times \Omega_{ \pm}\right),
$$

where $(+),(-)$ denote positrons, electrons, respectively, and

$$
\Omega_{ \pm} \equiv \boldsymbol{B} \pm \mu_{ \pm} \nabla \times \gamma_{ \pm} \boldsymbol{V}_{ \pm}
$$

is the generalized vorticity. Here velocities have been normalized to speed of light $c$, lengths to $c / \bar{\omega}_{c}$, time to $\bar{\omega}_{c}$ and magnetic field to an uniform ambient field $B_{o}$. With the following definitions: $\gamma_{ \pm}=\left(1-\left|\boldsymbol{V}_{ \pm}\right|^{2}\right)^{-1 / 2}, \mu_{ \pm} \equiv \bar{m}_{ \pm} /\left(\bar{m}_{+}+\bar{m}_{-}\right)$, $\bar{\omega}_{c}=e B_{o} / c\left(\bar{m}_{+}+\bar{m}_{-}\right), \quad \bar{\omega}_{p}^{2}=4 \pi n_{o} e^{2} /\left(\bar{m}_{+}+\bar{m}_{-}\right), \quad n_{+}=n_{-} \equiv n_{o} ;$ where $e$ is the electron's charge magnitude, $n_{o}$ is the uniform density in each beam's rest frame. Here $\mu$ plays the role of a reduced mass, where $\bar{m}_{ \pm}=m_{o} G\left(z_{ \pm}\right)$is an "effective mass" that depends on the temperature trough the function $G\left(z_{ \pm}\right)$ $=K_{3}\left(z_{ \pm}\right) / K_{2}\left(z_{ \pm}\right)$with argument $z_{ \pm}=m_{o} / T_{ \pm} ; m_{o}$ and $T_{ \pm}$are, respectively, the invariant rest mass and temperature for both species. For low temperatures $\left(z_{ \pm} \gg 1, T_{ \pm} \ll m_{o}\right) G\left(z_{ \pm}\right) \approx 1$ $+(5 / 2) T_{ \pm} / m_{o}$ then $\bar{m}_{ \pm} \approx m_{o}+(5 / 2) T_{ \pm}$, in a cold plasma $\bar{m}_{ \pm}=m_{o}$. For high (relativistic) temperatures $\left(z_{ \pm} \ll 1, T_{ \pm}\right.$ $\left.\gg m_{o}\right) G\left(z_{ \pm}\right) \approx 4 T_{ \pm} / m_{o}$ therefore $\bar{m}_{ \pm} \approx 4 T_{ \pm} \gg m_{o}$. Equation (6) represent a system of two equations with three unknowns. The system is completed by using the curl of Ampere's Law which in our normalizations becomes

$$
\boldsymbol{\nabla} \times(\boldsymbol{\nabla} \times \boldsymbol{B})+\frac{\partial^{2} \boldsymbol{B}}{\partial t^{2}}=\frac{c^{2}}{\bar{V}_{A}^{2}} \boldsymbol{\nabla} \times\left[\gamma_{+} \boldsymbol{V}_{+}-\gamma_{-} \boldsymbol{V}_{-}\right]
$$

where $\bar{V}_{A}=B_{o} / \sqrt{4 \pi n_{o}\left(\bar{m}_{+}+\bar{m}_{-}\right)}$. Note that in Eq. (8) the vector part, $\boldsymbol{j}=e n_{o}\left(\gamma_{+} \boldsymbol{V}_{+}-\gamma_{-} \boldsymbol{V}_{-}\right)$, of the four current has been used. Which makes it valid in both frames: CM and each beam's (of the pair) frame. From now on we assume that the equilibrium temperatures are equal $T_{+}=T_{-}=T$, and we call $G(z)$ simply $G(T)$. With temperatures assumed to be equal, the reduced mass becomes $\mu_{+}=\mu_{-}=1 / 2$. Note that this does not mean the temperature effects have disappeared, for $G(T)$ is still embedded in every $\bar{m}$ term.

\section{RELATIVISTIC WAVES}

In this paper, we investigate linear waves in a region far from the surface of the neutron star, such that, the magnetic field $B$ is still strong, but less than the critical value $B_{c r}$ $=\left(m_{e}^{2} c^{3} / e \hbar\right) \approx 4.4 \times 10^{13} \mathrm{G}$ so that all quantum effects can be ignored. Also, the curvature of the pulsar magnetic field, can be neglected for wave propagation at wavelengths satisfying $\lambda \ll B /(\partial B / \partial r)$, that is, when the wavelength is much less than the scale length of the magnetic field inhomogeneity. Such a system could be studied in Cartesian coordinates. One, of course, must make sure that the e-p plasmas are sufficiently long lived for the collective effects to be observed. It has been shown in Refs. $[18,19]$ that annihilation rates for electron-positrons (or positronium bound state formations) are much longer than the characteristic scale times for collective oscillations, typical plasma frequencies (at densities of interest), such that the pair plasma will live sufficiently long for many collective oscillations to take place. Dissipation effects induced by collisions can also be neglected due to the smallness of the e-p (or e-e) scattering cross section $\sigma \sim\left(e^{2} / E\right)^{2} \sim 10^{-30} \mathrm{~cm}^{2}$ (at relativistic energies in the range $\left.\gamma \sim 10-10^{2}\right)$. It should be pointed out different terminologies used in the literature to describe waves in e-p plasmas can create confusion see, e.g., [20]. Here we 
will use the conventional language used to describe the electron-ion plasmas.

To proceed we will use the index $\alpha$ to represent the particle species, which in our case takes only two values: positive or negative. Using a standard vector identity we can write Eq. (6) as

$$
\partial_{t} \Omega_{\alpha}=-\Omega_{\alpha}\left(\boldsymbol{\nabla} \cdot \boldsymbol{V}_{\alpha}\right)+\left(\Omega_{\alpha} \cdot \boldsymbol{\nabla}\right) \boldsymbol{V}_{\alpha}-\left(\boldsymbol{V}_{\alpha} \cdot \boldsymbol{\nabla}\right) \Omega_{\alpha} .
$$

$\left(\nabla \cdot \Omega_{\alpha}=0\right)$. We take the equilibrium fluid velocities in the $\mathrm{CM}$ frame (or equivalently in a frame in which they are equal) to be $\boldsymbol{V}_{+}=-\boldsymbol{V}_{o}, \boldsymbol{V}_{-}=\boldsymbol{V}_{o}$ where $\boldsymbol{V}_{o}=V_{o} \hat{z}$ ( $V_{o}$ a constant), so electrons (positrons) move with positive (negative) velocity along $\hat{z}$. Note that counterstreaming pair plasmas are gyrotropic [9], thus the modes are elliptically polarized in general. To study linear waves, we expand

$$
\begin{gathered}
\boldsymbol{V}_{\alpha}=-\alpha \boldsymbol{V}_{o}+\boldsymbol{v}_{\alpha}, \\
\boldsymbol{B}=\hat{z}+\boldsymbol{b},
\end{gathered}
$$

where $\hat{z}$ is a normalized (to $B_{o}$ ) equilibrium field. Equation (10) leads to

$$
\gamma_{\alpha}=\gamma_{o}-\alpha \gamma_{o}^{3} \boldsymbol{V}_{o} \cdot \boldsymbol{v}_{\alpha}
$$

where $\gamma_{o}=\left(1-V_{o}^{2}\right)^{-1 / 2}=$ constant. Using Eqs. (10)-(12) in Eq. (7) we find

$$
\Omega_{\alpha}=\hat{z}+\Omega_{\alpha}^{\prime},
$$

where

$$
\Omega_{\alpha}^{\prime}=\boldsymbol{b}+\frac{\alpha}{2} \boldsymbol{\nabla} \times\left[\gamma_{o} \boldsymbol{v}_{\alpha}+\gamma_{o}^{3}\left(\boldsymbol{V}_{o} \cdot \boldsymbol{v}_{\alpha}\right) \boldsymbol{V}_{o}\right]
$$

is the perturbed vorticity. Then using Eqs. (10) and (14) and $\boldsymbol{\nabla} \cdot \boldsymbol{v}_{\alpha}=0$ we can linearize Eq. (9) to get

$$
\left(\partial_{t}-\alpha \boldsymbol{V}_{o} \cdot \nabla\right) \Omega_{\alpha}^{\prime}=(\hat{z} \cdot \nabla) \boldsymbol{v}_{\alpha} .
$$

The system is closed with Eq. (8) which, after linearization, becomes

$$
-\nabla^{2} \boldsymbol{b}+\partial_{t}^{2} \boldsymbol{b}=\frac{1}{d^{2}} \sum_{\alpha} \alpha \boldsymbol{\nabla} \times\left[\gamma_{o} \boldsymbol{v}_{\alpha}+\gamma_{o}^{3}\left(\boldsymbol{V}_{o} \cdot \boldsymbol{v}_{\alpha}\right) \boldsymbol{V}_{o}\right]
$$

where $d \equiv \bar{V}_{A} / c=\bar{\omega}_{c} / \bar{\omega}_{p}=\omega_{c} /\left[\sqrt{G} \omega_{p}\right]$.

We solve the system of linearized equations by assuming that the perturbative quantities, $\boldsymbol{b}, \boldsymbol{v}_{\alpha}$ vary like $e^{i(\boldsymbol{k} \cdot \boldsymbol{r}-\omega t)}$. We use Cartesian coordinates with the $\hat{z}$ axis along the ambient magnetic field and the wave vector $\boldsymbol{k}=k(\sin \theta, 0, \cos \theta)$ in the $\hat{x}-\hat{z}$ plane. We also drop the primes from the perturbed vorticities to simplify the notation.

With these assumptions Eqs. (15) and (16) become

$$
\begin{aligned}
& \left(\omega+\alpha V_{o} k \cos \theta\right) \Omega_{x}^{\alpha}=-k \cos \theta v_{x}^{\alpha}, \\
& \left(\omega+\alpha V_{o} k \cos \theta\right) \Omega_{y}^{\alpha}=-k \cos \theta v_{y}^{\alpha}, \\
& \left(\omega+\alpha V_{o} k \cos \theta\right) \Omega_{z}^{\alpha}=-k \cos \theta v_{z}^{\alpha},
\end{aligned}
$$

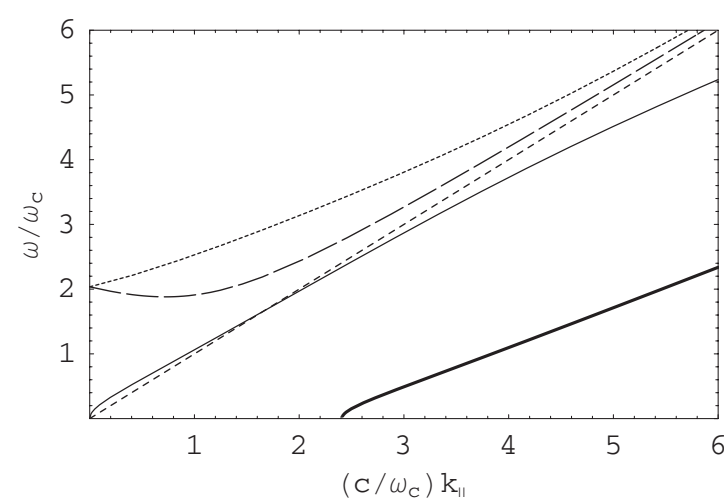

FIG. 1. Dispersion curves for exactly parallel propagation $(T$ $\left.\ll m_{o}\right)$. There are four modes, the intermediate-dashed line is the vacuum dispersion $\omega=k c$. For the slow Alfvén mode (thick solid line) $k<2 / V_{o} \gamma_{o}$ denotes the region of the cyclotron two-stream instability. The EMP modes (long and short dashed lines) have cutoff at $\omega=\sqrt{4 \omega_{p}^{2} / G+4 \omega_{c}^{2} / G^{2} \gamma_{o}^{2}}$. The point $k>2 \sqrt{\gamma_{o}^{2}-1}$ is where the fast Alfvén mode (continues line) becomes subluminous. The numerical parameters $\gamma_{o}=1.3, d=1.5$ where chosen for the sake of graphical clarity.

$$
\left(k^{2}-\omega^{2}\right) b_{x}=\frac{1}{d^{2}}\left[-i \gamma_{o} k \cos \theta\left(v_{y}^{+}-v_{y}^{-}\right)\right]
$$

$$
\left(k^{2}-\omega^{2}\right) b_{y}=\frac{1}{d^{2}}\left[i \gamma_{o} k \cos \theta\left(v_{x}^{+}-v_{x}^{-}\right)-i \gamma_{o}^{3} k \sin \theta\left(v_{z}^{+}-v_{z}^{-}\right)\right]
$$

$$
\left(k^{2}-\omega^{2}\right) b_{z}=\frac{1}{d^{2}}\left[i \gamma_{o} k \sin \theta\left(v_{y}^{+}-v_{y}^{-}\right)\right]
$$

where

$$
\Omega_{x}^{\alpha}=b_{x}+\frac{\alpha}{2}\left[-i \gamma_{o} k \cos \theta v_{y}^{\alpha}\right]
$$

$$
\begin{gathered}
\Omega_{y}^{\alpha}=b_{y}+\frac{\alpha}{2}\left[i \gamma_{o} k \cos \theta v_{x}^{\alpha}-i \gamma_{o}^{3} k \sin \theta v_{z}^{\alpha}\right], \\
\Omega_{z}^{\alpha}=b_{z}+\frac{\alpha}{2}\left[i \gamma_{o} k \sin \theta v_{y}^{\alpha}\right] .
\end{gathered}
$$

The system of Eqs. (17) and (18) consists of nine equations and nine unknowns. However they are not independent since we have used $\boldsymbol{\nabla} \cdot \boldsymbol{v}=0$ (together with $\boldsymbol{\nabla} \cdot \boldsymbol{b}=0$ ). In fact it is not difficult to see that the set of Eqs. (17a), (17b), (18a), and (18b) represent all the independent equations. This set is reducible to the matrix 


$$
\left(\begin{array}{cccc}
\frac{1}{\omega_{D}^{+}} & 0 & -i \gamma_{o} P & i \frac{\gamma_{o}}{L} \\
0 & \frac{1}{\omega_{D}^{-}} & -i \frac{\gamma_{o}}{L} & i \gamma_{o} P \\
i \gamma_{o}\left[\cos ^{2} \theta+\gamma_{o}^{2} \sin ^{2} \theta\right] P & -i \frac{\gamma_{o}}{L}\left[\cos ^{2} \theta+\gamma_{o}^{2} \sin ^{2} \theta\right] & \frac{\cos ^{2} \theta}{\omega_{D}^{+}} & 0 \\
i \frac{\gamma_{o}}{L}\left[\cos ^{2} \theta+\gamma_{o}^{2} \sin ^{2} \theta\right] & -i \gamma_{o}\left[\cos ^{2} \theta+\gamma_{o}^{2} \sin ^{2} \theta\right] P & 0 & \frac{\cos ^{2} \theta}{\omega_{D}^{-}}
\end{array}\right)\left(\begin{array}{c}
v_{x}^{+} \\
v_{x}^{-} \\
v_{y}^{+} \\
v_{y}^{-}
\end{array}\right)=0,
$$

where we have used the following abbreviations to simplify the notation: $L \equiv d^{2}\left(k^{2}-\omega^{2}\right), P \equiv 1 / L+1 / 2, \quad \omega_{D}^{ \pm}$ $\equiv \omega \pm V_{o} k \cos \theta$.

\section{A. Parallel propagation $(k \| \hat{z})$}

When wave propagation is along the magnetic field $(\theta$ $=0$ ), the determinant of Eq. (22) gives the dispersion relation

$$
\begin{aligned}
0= & +\frac{2 \gamma_{o}^{2}}{L^{2}}\left(\omega^{2}-k^{2} V_{o}^{2}\right)-2 \gamma_{o}^{2}\left(\omega^{2}+k^{2} V_{o}^{2}\right)\left(\frac{1}{2}+\frac{1}{L}\right)^{2} \\
& +\gamma_{o}^{4}\left(\omega-k V_{o}\right)^{2}\left(\omega+k V_{o}\right)^{2}\left[\left(\frac{1}{2}+\frac{1}{L}\right)^{2}-\frac{1}{L^{2}}\right]^{2} .
\end{aligned}
$$

Equation (23) gives us four transverse modes. The frequencies of the first two transverse modes are

$$
\begin{aligned}
\omega\left(\begin{array}{l}
1 \\
2
\end{array}\right)= & {\left[\frac{2}{d^{2}}+\frac{2}{\gamma_{o}^{2}} \mp \frac{2 V_{o} k}{\gamma_{o}}+\left(1+V_{o}^{2}\right) \frac{k^{2}}{2}+\frac{1}{2 \gamma_{o}^{2} d^{2}}\right.} \\
& \times\left\{\left[4 \gamma_{o}^{2}+4 d^{2} \mp 4 \gamma_{o} V_{o} d^{2} k+\gamma_{o}^{2} d^{2}\left(1+V_{o}^{2}\right) k^{2}\right]^{2}\right. \\
& \left.\left.-4 \gamma_{o}^{2} d^{2} \Upsilon_{\mp}\right\}^{1 / 2}\right]^{1 / 2} .
\end{aligned}
$$

Where

$$
\Upsilon_{\mp}=\mp 8 \gamma_{o} V_{o} k+4\left(\gamma_{o}^{2} V_{o}^{2}+d^{2}\right) k^{2} \mp 4 \gamma_{o} V_{o} d^{2} k^{3}+\gamma_{o}^{2} V_{o}^{2} d^{2} k^{4} \text {. }
$$

These frequencies are degenerate electromagnetic plasma (EMP) modes (see Fig. 1), becoming asymptotic to $k c$ at large $k$. Both modes are superluminous $\left(V_{\phi}=\omega / k>c\right)$; however solution one, corresponding to the upper signs, is slower than solution two. Both modes have cutoff ( $k \rightarrow 0$ limit) at

$$
\omega^{2}=\frac{4}{d^{2}}+\frac{4}{\gamma_{o}^{2}}=\frac{4 \bar{\omega}_{p}^{2}}{\bar{\omega}_{c}^{2}}+\frac{4}{\gamma_{o}^{2}}
$$

or in physical units,

$$
\omega^{2}=4 \frac{\omega_{p}^{2}}{G(T)}+4 \frac{\omega_{c}^{2}}{\gamma_{o}^{2} G(T)^{2}}
$$

the hybrid frequency. We have made the temperature explicit in Eq. (26). Note that $\bar{\omega}_{p}^{2}=\omega_{p}^{2} / G$ where $\omega_{p}^{2}=\left(4 \pi n_{o} e^{2} / 2 m_{o}\right)$; likewise $\bar{\omega}_{c}=\omega_{c} / G$ where $\omega_{c}=\left(e B_{o} / c 2 m_{o}\right)$. Equation (26) is our first important result. Note how the temperature affects the cutoff; for example, for ultra relativistic high temperatures $\left(T \gg m_{o}\right)$ the cutoff decreases. Since $T \gg m_{o}$ implies $G$ $\approx 4 T / m_{o}$, then the effective mass becomes $\bar{m} \approx 4 T \gg m_{o}$. Therefore the fluid inertia is primarily provided by the random thermal motion of the particles. If, on the other hand, $T \ll m_{o}, G(T) \approx 1$, we recover the cold plasma cutoff [9]

$$
\omega^{2}=4 \omega_{p}^{2}+4 \frac{\omega_{c}^{2}}{\gamma_{o}^{2}} .
$$

In the large $k$ limit, however, these EMP waves [Eq. (24)] become

$$
\omega \approx\left[\mp \frac{2 V_{o} k}{\gamma_{o}}+\left(1+V_{o}^{2}\right) \frac{k^{2}}{2}+\frac{k^{2}}{2}\left(1-V_{o}^{2}\right) \pm \frac{2 V_{o} k}{\gamma_{o}}\right]^{1 / 2} \approx k,
$$

the vacuum dispersion relation. In physical units Eq. (28) is

$$
\frac{\omega}{\bar{\omega}_{c}} \approx k \frac{c}{\bar{\omega}_{c}} \Rightarrow \omega=k c .
$$

Thus in this large $k$ limit temperature effects are negligible. Therefore these modes are affected by temperature in the range of long wavelengths only. As you go from cold to hot plasma the modes go from EMP to light waves; see Figs. 2 and 3. Particularly for secondary plasmas in pulsars $T$ $\sim m_{o}[G(1) \approx 4.37]$ the degeneracy of the EMP modes is still present see Fig. 2.

The other two transverse modes are

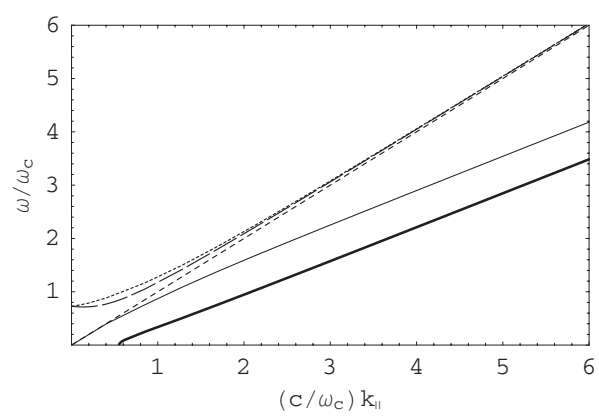

FIG. 2. Dispersion curves for parallel propagation, for temperatures $T=m_{o}(G=4.37)$. The labeling is the same used in Fig. 1. Note that, at short $k$, the degeneracy of the EMP modes is still present, and the slow Alfvén wave is still unstable. 


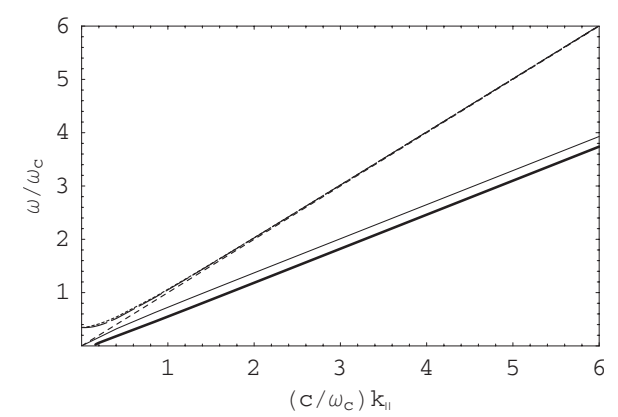

FIG. 3. Dispersion curves for parallel propagation, for still higher temperatures $T>m_{o}$. The labeling is the same used in Fig. 1. Note that the EMP modes have virtually become light waves.

$$
\begin{aligned}
\omega\left(\begin{array}{l}
3 \\
4
\end{array}\right)= & \frac{1}{\sqrt{2}}\left[\frac{4}{\gamma_{o}^{2}}+\frac{4}{d^{2}} \mp \frac{4 V_{o} k}{\gamma_{o}}+k^{2}\left(1+V_{o}^{2}\right)-\frac{1}{\gamma_{o}^{2} d^{2}}\right. \\
& \times\left\{\left[4 \gamma_{o}^{2}+4 d^{2} \mp 4 \gamma_{o} V_{o} d^{2} k+\gamma_{o}^{2} d^{2}\left(1+V_{o}^{2}\right) k^{2}\right]^{2}\right. \\
& \left.\left.-4 \gamma_{o}^{2} d^{2} \Upsilon_{\mp}\right\}^{1 / 2}\right]^{1 / 2} .
\end{aligned}
$$

Solution three, corresponding to the upper signs, will be called (see Sec. III B) the slow Alfvén wave; solution number four will be called fast Alfvén wave (see Fig. 1). Of the two Alfvén waves, the fast mode is superluminous (for small wave vectors) becoming subluminous at $k c$ $>2\left(\omega_{c} / G\right) \sqrt{\gamma_{o}^{2}-1}$. The slow mode is always subluminous and is cyclotron two-stream unstable for small wave vectors. In fact there are two unstable regions for this slow wave. The frequency vanishes at

$$
k_{ \pm}=\frac{1}{\gamma_{o} V_{o} G} \pm \frac{1}{\gamma_{o} V_{o} G}\left(1-4 \frac{\gamma_{o}^{2} V_{o}^{2} G \omega_{p}^{2}}{\omega_{c}^{2}}\right)^{1 / 2}
$$

and

$$
k_{c}=\frac{2}{\gamma_{o} V_{o}} .
$$

Thus the wave is unstable in the regions $0<k<k_{-}$and $k_{+}$ $<k<k_{c}$, (see Fig. 4). If $G \approx 1$ we recover the cold limit

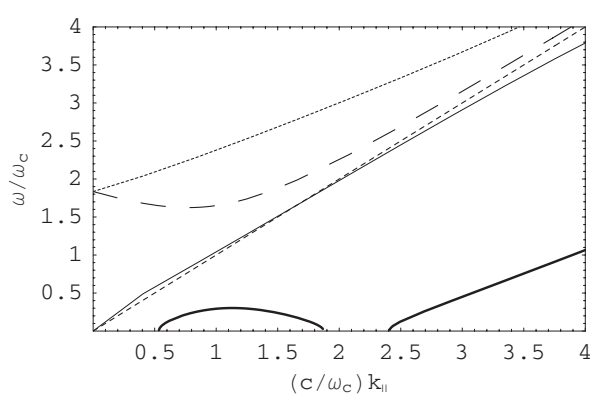

FIG. 4. Dispersion curves for exactly parallel propagation with ratio $4 \gamma_{o}^{2} V_{o}^{2} / d^{2}<1$. Note that, as explained in text, the slow Alfvén wave (thick solid line) is unstable in two regions: $0<k<k_{-}$ $\approx 0.53$ and $k_{+} \approx 1.87<k<k_{c} \approx 2.41$. The labeling for the rest of the curves is the same used in Fig. 1. The numerical parameters $\gamma_{o}$ $=1.3, d=2$ where chosen for the sake of graphical clarity. result for $k_{ \pm}$[9]. Depending on the ratio $\left(4 \gamma_{o}^{2} V_{o}^{2} G \omega_{p}^{2} / \omega_{c}^{2}\right), k_{-}$ and $k_{+}$can merge to form a sole region of instability $0<k$ $<k_{c}$ (see Fig. 1).

To understand better $k_{c}$ we find an approximate analytical expression by taking the small $k$ limit of Eq. (30). After some algebra we find

$$
\omega\left(\begin{array}{l}
3 \\
4
\end{array}\right) \approx \sqrt{\frac{\mp 2 V_{o} \gamma_{o} k+k^{2}\left(d^{2} / \gamma_{o}^{2}+\gamma_{o}^{2} V_{o}^{2}\right)}{\gamma_{o}^{2}+d^{2}}} .
$$

Clearly solution three, the slow Alfvén wave, becomes unstable for

$$
k<\frac{2 V_{o} \gamma_{o}}{\left(\gamma_{o}^{2} V_{o}^{2}+d^{2} / \gamma_{o}^{2}\right)} .
$$

If $\gamma_{o}^{2} V_{o}^{2} \gg d^{2} / \gamma_{o}^{2}$, then this condition becomes

$$
k<\frac{2}{\gamma_{o} V_{o}}
$$

or in physical units

$$
k V_{o}<2 \frac{\bar{\omega}_{c}}{\gamma_{o}}=2 \frac{\omega_{c}}{G(T) \gamma_{o}} .
$$

Note how the temperature is manifested. For low temperatures $G(T) \approx 1$ we recover the cold relativistic limit $k V_{o}$ $<2 \omega_{c} / \gamma_{o}$ [9]. Thus we may call this condition relativistic temperature-dependent cyclotron two-stream instability.

On the other hand, if $d^{2} / \gamma_{o}^{2} \gg V_{o}^{2} \gamma_{o}^{2}$, which is a more appropriate limit for pulsar plasmas, then Eq. (34) gives in physical units

$$
k c<2 \frac{V_{o}}{c} \gamma_{o}^{3} \frac{\bar{\omega}_{p}^{2}}{\bar{\omega}_{c}}=2 \frac{V_{o}}{c} \gamma_{o}^{3} \frac{\omega_{p}^{2}}{\omega_{c}} .
$$

Therefore in this particular limit the criterion of instability is valid in both cold and very high temperature plasmas. The maximum growth rate for this instability (in the limit $\left.d^{2} / \gamma_{o}^{2} \gg V_{o}^{2} \gamma_{o}^{2}\right)$ is

$$
\operatorname{Im}(\omega)_{\max } \approx \frac{V_{o}}{c} \gamma_{o}^{2} \frac{\omega_{p}^{2}}{\omega_{c}} .
$$

Thus it is not suppressed by temperature effects (Fig. 5).

At large $k$, the Alfvénic modes, Eq. (30) are asymptotic to

$$
\omega \approx k V_{o} \mp \frac{2}{\gamma_{o}},
$$

see Figs. 1-3, or in physical units to

$$
\omega \approx k V_{o} \mp \frac{2 \omega_{c}}{G(T) \gamma_{o}} .
$$

Revealing temperature modifications to the cold limit result. We may call these relativistic temperature-dependent cyclotron waves.

\section{B. Zero streaming limit $\left(V_{o} \rightarrow 0\right)$}

If we go to the limit of no streaming (see Fig. 6), then the degeneracy of EMP modes disappears. Equations (26) and 


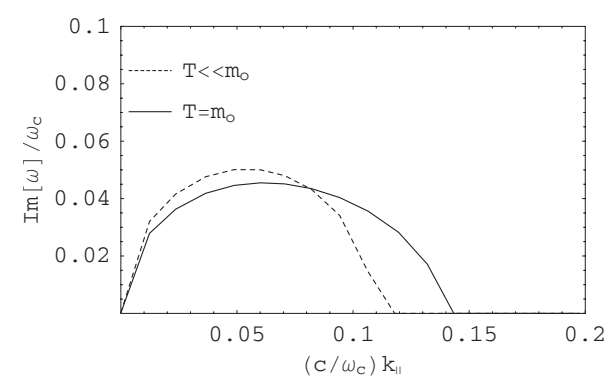

FIG. 5. Numerical growth rate curves of the slow Alfvén mode, for various temperatures. Limit $d^{2} / \gamma_{o}^{2} \gg V_{o}^{2} \gamma_{o}^{2}$. Note that, as explained in the text, for temperatures of the order of $T \sim m_{o}$ the instability is not completely suppressed. With numerical parameters $\gamma_{o}=1.3, d=4$.

(29) remain valid but with $\gamma_{o} \rightarrow 1$. Note that the temperature effects still can bring down the cutoff as shown in Fig. 6.

On the other hand, for the Alfvénic modes, we find from Eq. (33)

$$
\omega \approx \frac{k d}{\left(1+d^{2}\right)^{1 / 2}}
$$

or in physical units

$$
\omega \approx \frac{k \bar{V}_{A}}{\left(1+\bar{V}_{A}^{2} / c^{2}\right)^{1 / 2}}
$$

the Alfvénic dispersion. This is why we called solutions three and four slow and fast Alfvénic modes. Note that in this limit the degeneracy is lost. Yet temperature effects (in the limit $\left.\bar{V}_{A}<c\right)$ can bring down the frequency, [remember $\bar{V}_{A}$ $\left.=V_{A} / \sqrt{G(T)}\right]$; see Fig. 6 .

In the large $k$ limit, these Alfvénic modes [see Eq. (38)], are asymptotic to

$$
\omega=\mp 2 \bar{\omega}_{c}=\mp 2 \frac{\omega_{c}}{G(T)},
$$

the cyclotron frequency branch (see Fig. 6).

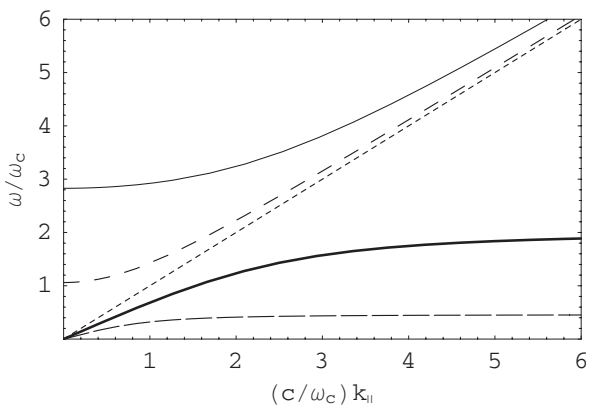

FIG. 6. Dispersion curves for zero streaming $\left(\gamma_{o}=1, d=1\right)$. For temperatures: $T \ll m_{o}$ (continues lines) and $T=m_{o}$ (dashed lines). Notice the lowered cutoff of the EMP modes, and the frequency drop for the Alfvénic mode at $T=m_{o}$.

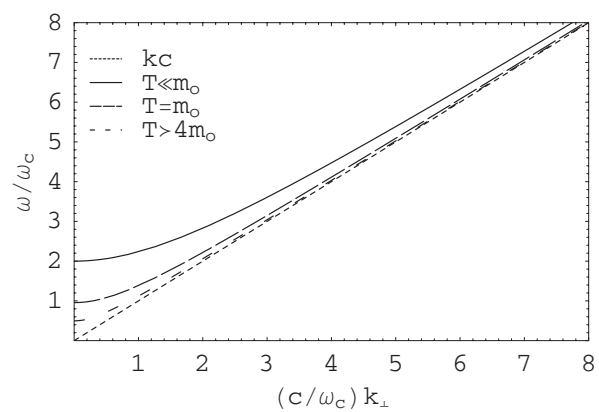

FIG. 7. Dispersion curves for perpendicular propagation $(\theta$ $=\pi / 2, d=1)$. Note how the increasing of temperature lowers the plasma cutoff until it reaches the asymptotic $k c$ limit.

\section{Perpendicular propagation $(k \perp \hat{z})$}

For propagation perpendicular to the magnetic field $(\theta$ $=\pi / 2$ ), Eq. (22) gives the dispersion relation

$$
\left(\frac{1}{2}+\frac{1}{L}\right)^{2}-\frac{1}{L^{2}}=0
$$

for $L=d^{2}\left(k^{2}-\omega^{2}\right) \neq 0$. Its solution is

$$
\omega^{2}=k^{2}+\frac{4}{d^{2}}
$$

or in physical units

$$
\omega^{2}=k^{2} c^{2}+\frac{4 \omega_{p}^{2}}{G(T)}
$$

the ordinary mode. Note that as the temperature increases the ordinary mode becomes a light wave (see Fig. 7). The fact that only the ordinary mode is present for $\theta=\pi / 2$, is explained by looking at Eq. (15). They become

$$
\omega \Omega_{\alpha}^{\prime}=0 \Rightarrow \Omega_{\alpha}^{\prime}=0,
$$

therefore $\boldsymbol{b}=-(\alpha / 2) \boldsymbol{\nabla}_{\perp} \times\left[\gamma_{o} \boldsymbol{v}_{\alpha}+\gamma_{o}^{3}\left(\boldsymbol{V}_{o} \cdot \boldsymbol{v}_{\alpha}\right) \boldsymbol{V}_{o}\right]$, which, after substitution in Eq. (16) gives

$$
\left(-\nabla_{\perp}^{2}+\partial_{t}^{2}\right) \boldsymbol{b}=-\frac{4}{d^{2}} \boldsymbol{b} \Rightarrow \omega^{2}=k^{2}+\frac{4}{d^{2}}
$$

after Fourier transform. The fact the perturbed vorticity is zero $\Omega_{\alpha}^{\prime}=0$ makes the magnetic field antiparallel with the sum of the vorticities (the curl of the current). Therefore the particles do not feel the ambient magnetic field.

\section{Super strong magnetic field $(\theta=0)$}

For strong magnetic fields (but less than $B_{c r}$ ) and mildly relativistic velocities $\left(\gamma_{o}=4\right)$ the parallel curves $(\theta=0)$ are shown in Figs. 8-10. The modes appear as straight lines. To understand this, we take the $d \rightarrow \infty$, limit of Eq. (24), the EMP modes. After some algebra we find

$$
\omega\left(\begin{array}{l}
1 \\
2
\end{array}\right)=\frac{1}{G}\left[\frac{4}{\gamma_{o}^{2}} \mp \frac{4 V_{o}}{\gamma_{o}} G k+V_{o}^{2} G^{2} k^{2}\right]^{1 / 2} .
$$

Where $G(T)$ has been written explicitly. It is not difficult to see that for small or large $k, \omega_{2}$ always gives 


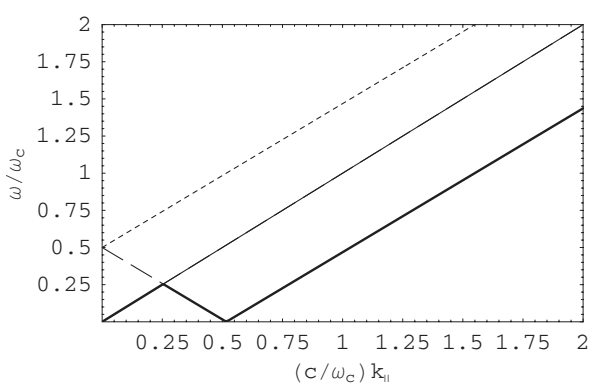

FIG. 8. Dispersion curves for parallel propagation. Super strong B field, cold plasma. The fast EMP mode (short dashed line) have dispersion $\omega \approx 2 / \gamma_{o}+V_{o} k$. The slow Alfvén mode (thick solid line) is divided in three regions. The slow EMP (long dashed line) and fast Alfvén modes (continues line) have dispersion relations as described in the text. The numerical parameters $\gamma_{o}=4, d=10^{5}$ were chosen for the sake of graphical clarity.

$$
\omega_{2} \approx \frac{2}{\gamma_{o} G}+V_{o} k .
$$

For low temperatures $(G \approx 1)$ the cutoff (with the numerical values given to the plot) is at $2 / \gamma_{o}=0.5$, as shown in Fig. 8 . Solution one on the other hand gives in the small $k$ limit

$$
\omega_{1} \approx \frac{2}{\gamma_{o} G}-V_{o} k,
$$

i.e., same cutoff but negative slope (see Fig. 8). For large $k$ it gives $\omega_{1} \approx V_{o} k$. The other two transverse modes Eq. (30) (Alfvénic) have the following limits: solution four gives $\omega_{4}$ $\approx k$ (for large and small $k$ ) and solution three (the slow Alfvénic) gives

$$
\omega_{3} \approx \begin{cases}k & \text { if } k \lesssim 0.25 \\ \frac{2}{\gamma_{o} G}-V_{o} k & \text { if } 0.25<k \lesssim 0.5 \\ -\frac{2}{\gamma_{o} G}+V_{o} k & \text { if } k \gtrsim 0.5\end{cases}
$$

Note that temperature effects, for all four modes in a strong magnetic field, are manifested trough the terms $\pm 2 /\left[\gamma_{o} G(T)\right]$. Thus as temperature rises both cutoffs (negative and positive) approach zero. Therefore the modes ap-

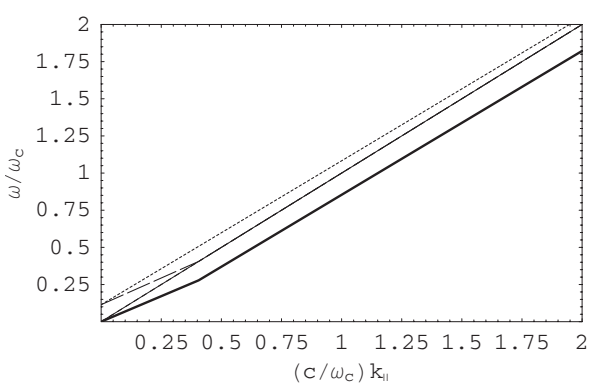

FIG. 9. Dispersion curves for Parallel propagation. Strong B field. For $T=m_{o}, \gamma_{o}=4, d=10^{5}$. Notice the increase of temperature manifested through the decreasing cutoffs, making the curves approach the line $k c$. Labeling same as in Fig. 8.

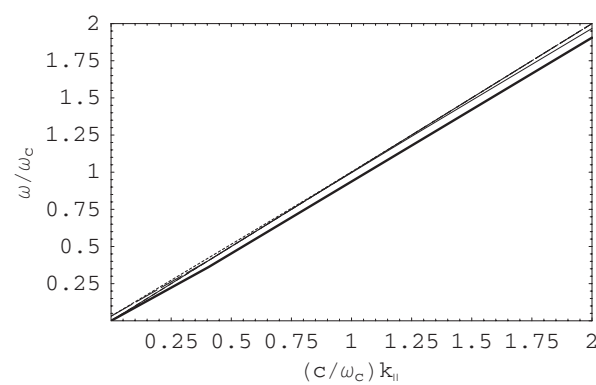

FIG. 10. Dispersion for parallel propagation, for still higher temperatures $T>m_{o}$. Strong B field. Still $\gamma_{o}=4, d=10^{5}$. Here the cutoffs $\pm 2 /\left[\gamma_{o} G(T)\right]$, have become approximately zero and the curves have become virtually the line $k$. Labeling same as in Fig. 8.

proach $k$ (see Figs. 9 and 10 ). If $G \approx 1$ we recover the cold plasma limit [9], as shown in Fig. 8. Note that the line along $k$ ( $k c$ in physical units) is thus various lines very close together.

\section{E. Oblique propagation $(\theta=\pi / 4)$}

For oblique propagation the dispersion relation is cumbersome, however the modes are similar to the parallel propagation case. We will describe them qualitatively with the help of Fig. 11. There we see four curves, two of them are EMP waves (degenerate) just like in the parallel propagation case but with closer phase velocity. The other two are Alfvénic (or cyclotron in the large $k$ region) modes that split into a fast and slow mode.

The fast mode becomes subluminous at $k c$ $>2\left(\omega_{c} / G\right) \sqrt{2\left(\gamma_{o}^{2}-1\right) /\left(1+\gamma_{o}^{2}\right)^{3}}$, which happens faster than in the parallel propagation case. The slow Alfvénic mode is cyclotron two-stream unstable at large wavelengths, just like in the parallel case. As temperature increases to $T>m_{o}$ then we see that in fact the frequency decreases similarly to the parallel propagation case (see Fig. 12).

\section{F. Super strong magnetic field $(\theta=\pi / 4)$}

The case of oblique propagation with, $\gamma_{o}=10$ and strong magnetic field is shown in Fig. 13. There we see that the two

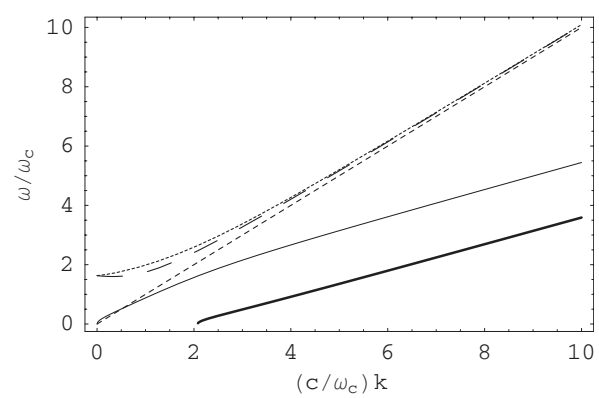

FIG. 11. Dispersion curves for oblique propagation. Here we have four modes, two of them are EMP modes (short and long dashed lines). The slow branches (continues and thick lines) are the fast and slow Alfvénic modes respectively. Cold plasma $\left(T \ll m_{o}\right)$, $\gamma_{o}=1.3, d=2$. 


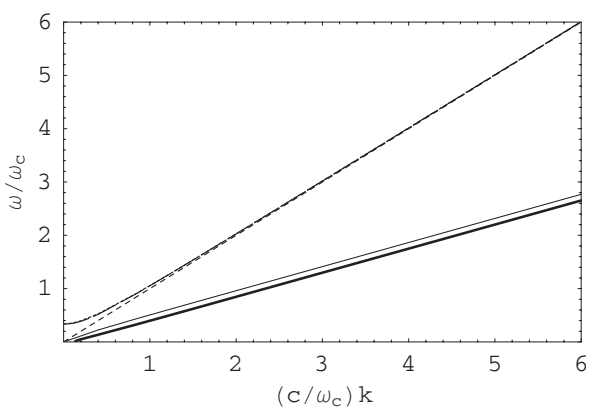

FIG. 12. Dispersion curves for oblique propagation, for high temperatures $T>m_{o}\left(\gamma_{o}=1.3, d=1.5\right)$. Labeling same as in Fig. 11.

EMP modes have become light waves and the Alfvén waves have become asymptoted to

$$
\omega \approx \frac{1}{\sqrt{2}} k c \sqrt{2-\left(V_{o} / c\right)^{2}} .
$$

Note first that temperature effects are negligible in this particular limit. Second note that these waves do not reach the asymptotic limit of $k c$ contrary to what happens for parallel propagation. Here the asymptotic value $\omega \approx k c / \sqrt{2}$ (when $\left.V_{o} \rightarrow c\right)$ is a geometric effect.

\section{NUMERICAL ESTIMATES}

In order to make numerical estimates, we will follow [6] with the full realization that parameters at the polar caps are model dependent - there are several models, for example, for the generation of secondary pairs, leading to considerable uncertainties in the estimates of plasma density.

Our reference plasma is created outside a pulsar with a period $P=0.2 \mathrm{~s}$, and magnetic (dipole) field strength $B_{o}=10^{12} \mathrm{G}$; the corresponding density of corotation and cyclotron frequency are: $n_{G J}=3.5 \times 10^{11} \mathrm{~cm}^{-3}, \omega_{c}=1.75$ $\times 10^{19} \mathrm{~s}^{-1}$. The pair plasma (in the pulsar frame) has a density of the order of $n_{ \pm}=\Gamma n_{G J}$ where $\Gamma \approx 10^{3}-10^{6}$ is the multiplicity factor [6]. Note that the multiplicity factor is uncertain (see, e.g., [10]). The primary beam has a density $n_{b}$ equal to $n_{G J}$ at the time of pair creation [13], and flows along the open magnetic lines with energies $\gamma_{b} \sim 10^{7}$. The energy of the pair plasma particles is in the range $\gamma_{p} \sim 10-10^{3}$.

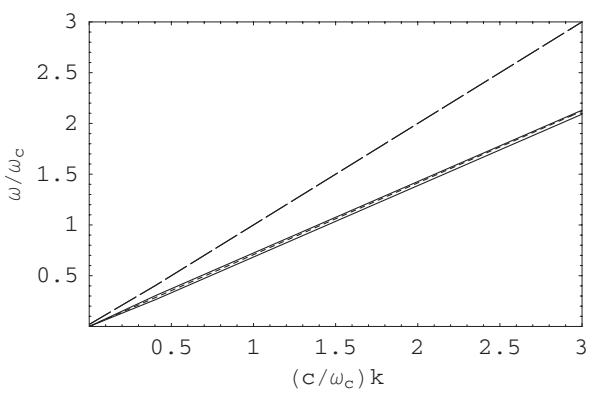

FIG. 13. Dispersion curves for Oblique propagation. Strong B field. The EMP modes (long dashed line) have become asymptoted to $k c$. The Alfvénic modes (continues and thick lines) have become asymptoted to $k c / \sqrt{2}$ (short dashed line). With $\gamma_{o}=10, d=10^{5}$.
The pair plasma rest frame densities (in each plasma beam) are related to the density $n_{G J}$ (measured in the pulsar frame) via the multiplicity factor $\Gamma$ and two Lorentz transformations: from the pulsar frame to the CM frame (with $\gamma_{p}$ ) and from the CM to each component's rest frame (with $\gamma_{o}$ ) that is $n_{o}=\Gamma n_{G J} / \gamma_{p} \gamma_{o}$ [6]. The corresponding plasma frequency (square of it) is $\omega_{p}^{2}=1.11 \times 10^{21}-10^{26} \mathrm{~s}^{-2}$.

For an instability to be a good candidate as a possible source of coherent radio emission generation, its growth rate, evaluated at the pulsar frame, should be much larger than the pulsar rotational frequency $w=2 \pi / P \approx 31.4 \mathrm{~s}^{-1}$, that is

$$
\frac{\operatorname{Im}(\omega)}{\gamma_{p}} \gg w
$$

where $\operatorname{Im}(\omega)=\left(V_{o} / c\right) \gamma_{o}^{2}\left(\omega_{p}^{2} / \omega_{c}\right)$. We can then calculate the growth rate of this instability by assuming typical relative streaming energies $\gamma_{o}$. (i) For mildly relativistic streaming $\gamma_{o}=10$, we find

$$
\operatorname{Im}(\omega) \approx 6.2 \times 10^{3}-10^{8} \mathrm{~s}^{-1} .
$$

(ii) For large relativistic streaming $\gamma_{o}=100$ we find

$$
\operatorname{Im}(\omega) \approx 6.3 \times 10^{5}-10^{10} \mathrm{~s}^{-1} .
$$

Therefore for case (i) and using $\gamma_{p}=10^{3}$, we find

$$
\frac{\operatorname{Im}(\omega)}{\gamma_{p}} \approx 6.2-10^{5} \mathrm{~s}^{-1},
$$

whereas for (ii)

$$
\frac{\operatorname{Im}(\omega)}{\gamma_{p}} \approx 6.3 \times 10^{2}-10^{7} \mathrm{~s}^{-1} \gg 31.4 \mathrm{~s}^{-1}
$$

We see from the above that, for case (i), lower limit only, the instability is not fast enough. The second case is more attractive of course, since it is evident that the instability has the potential of being a good candidate for exiting waves or particles that could eventually help a nonlinear process to produce coherent emission.

\section{CONCLUSIONS}

We have considered propagation of relativistic waves in streaming magnetized plasmas. For parallel propagation we have four modes.

(1) For finite temperature and finite magnetic field the stable fast Alfvén wave is superluminous for large wavelengths only, becoming subluminous at $k>2\left(\omega_{c} / c\right) \sqrt{\gamma_{o}^{2}-1}$. At large temperatures, however, it is always subluminous. In the super strong magnetic field case it has the vacuum dispersion relation.

(2) The slow Alfvén wave is cyclotron two-stream unstable for large wavelengths and always subluminous. For temperatures of the order of $T \sim m_{o}$ (and limit $\gamma_{o}^{2} V_{o}^{2} \gg d^{2} / \gamma_{o}^{2}$ ) it is unstable. For ultra high temperatures it can be stabilized. However in the limit $d^{2} / \gamma_{o}^{2} \gg V_{o}^{2} \gamma_{o}^{2}$ it is always unstable regardless of temperature. For strong magnetic field case it splits into three regions, with the dispersion relation $\omega=-2 /\left(\gamma_{o} G\right)+V_{o} k$ in the large $k$ region. 
(3) The other two modes are degenerate electromagnetic plasmas waves which at high temperature become light waves.

For oblique propagation with strong magnetic field our dispersion relation is unaffected by temperature: it is valid in both cold and/or hot magnetized plasmas.

Because our model is incompressible, we find only the ordinary mode for perpendicular propagation; neither the pure streaming instability nor the compressional wave is permitted. Most relevant to radio emission theories is our result that the slow Alfvén wave is unstable, and is not fully stabilized even at temperatures of the order of $T \sim m_{o}$. In addition, for typical values, the instability is quite fast and the waves can grow to such levels that the magnetic modulation could act as a wiggler with which the primary beam could interact simulating an FEL (free electron laser) like effect, yielding coherent radiation. Detailed description of this interesting "secondary" phenomenon will be presented elsewhere.

\section{ACKNOWLEDGMENTS}

The authors would like to thank Mel Oakes and anonymous referees for helpful comments and recommendations.
[1] M. A. Ruderman and P. G. Sutherland, Astrophys. J. 196, 51 (1975).

[2] S. M. Mahajan, G. Z. Machabeli, and A. D. Rogava, Astrophys. J. 479, L129 (1997).

[3] P. A. Sturrock, Astrophys. J. 164, 529 (1971).

[4] D. B. Melrose, Plasma Phys. Controlled Fusion 39, A93 (1997).

[5] J. Arons and J. J. Barnard, Astrophys. J. 302, 120 (1986).

[6] M. Lyutikov, Mon. Not. R. Astron. Soc. 293, 447 (1998).

[7] M. Gedalin, E. Gruman, and D. B. Melrose, Mon. Not. R. Astron. Soc. 325, 715 (2001).

[8] M. Gedalin, D. B. Melrose, and E. Gruman, Phys. Rev. E 57, 3399 (1998).

[9] M. W. Verdon and D. B. Melrose, Phys. Rev. E 77, 046403 (2008).

[10] P. N. Arendt, Jr. and J. A. Eilek, Astrophys. J. 581, 451 (2002).
[11] D. B. Melrose, J. Astrophys. Astron. 16, 137 (1995).

[12] P. Goldreich and W. H. Julian, Astrophys. J. 157, 869 (1969).

[13] A. F. Cheng and M. A. Ruderman, Astrophys. J. 212, 800 (1977).

[14] S. M. Mahajan, Phys. Rev. Lett. 90, 035001 (2003).

[15] V. I. Berezhiani, S. M. Mahajan, Z. Yoshida, and M. Ohhashi, Phys. Rev. E 65, 047402 (2002).

[16] V. I. Berezhiani and S. M. Mahajan, Phys. Rev. E 52, 1968 (1995).

[17] V. I. Berezhiani and S. M. Mahajan, Phys. Rev. Lett. 73, 1110 (1994).

[18] G. P. Zank and R. G. Greaves, Phys. Rev. E 51, 6079 (1995).

[19] N. Iwamoto, Phys. Rev. E 47, 604 (1993).

[20] D. Shapakidze, G. Machabeli, G. Melikidze, and D. Khechinashvili, Phys. Rev. E 67, 026407 (2003). 